\title{
Rosiglitazone Improves Insulin Sensitivity and Baroreflex Gain in Rats with Diet-Induced Obesity
}

\author{
Ding Zhao, Belinda H. McCully, and Virginia L. Brooks \\ Department of Physiology and Pharmacology, Oregon Health \& Science University, Portland, Oregon (D.Z., B.H.M., V.L.B.); \\ and School of Pharmacy, Hebei Medical University, Shijiazhuang, People's Republic of China (D.Z.)
}

Received March 23, 2012; accepted July 17, 2012

\begin{abstract}
Obesity decreases baroreflex gain (BRG); however, the mechanisms are unknown. We tested the hypothesis that impaired $B R G$ is related to the concurrent insulin resistance, and, therefore, BRG would be improved after treatment with the insulinsensitizing drug rosiglitazone. Male rats fed a high-fat diet diverged into obesity-prone (OP) and obesity-resistant (OR) groups after 2 weeks. Then, OP and OR rats, as well as control (CON) rats fed a standard diet, were treated daily for 2 to 3 weeks with rosiglitazone ( 3 or $6 \mathrm{mg} / \mathrm{kg}$ ) or its vehicle by gavage. Compared with OR and CON rats, conscious OP rats exhibited reductions in BRG $(\mathrm{OP}, 2.9 \pm 0.1 \mathrm{bpm} / \mathrm{mm} \mathrm{Hg}$; OR, $4.0 \pm 0.2$ $\mathrm{bpm} / \mathrm{mm} \mathrm{Hg}$; CON, $3.9 \pm 0.2 \mathrm{bpm} / \mathrm{mm} \mathrm{Hg} ; P<0.05)$ and insulin sensitivity (hyperinsulinemic euglycemic clamp; OP, $6.8 \pm 0.9 \mathrm{mg} / \mathrm{kg} \cdot \mathrm{min} ; \mathrm{OR}, 22.2 \pm 1.2 \mathrm{mg} / \mathrm{kg} \cdot \mathrm{min} ; \mathrm{CON}$, $17.7 \pm 0.8 \mathrm{mg} / \mathrm{kg} \cdot \mathrm{min} ; P<0.05)$, which were well correlated $\left(r^{2}=0.49 ; P<0.01\right)$. In OP rats, rosiglitazone dose-depend-
\end{abstract}

ently improved $(P<0.05)$ insulin sensitivity $(12.8 \pm 0.6 \mathrm{mg} / \mathrm{kg}$. $\mathrm{min}$ at $3 \mathrm{mg} / \mathrm{kg} ; 16.0 \pm 1.5 \mathrm{mg} / \mathrm{kg} \cdot \min$ at $6 \mathrm{mg} / \mathrm{kg})$ and $\mathrm{BRG}$ $(3.8 \pm 0.4 \mathrm{bpm} / \mathrm{mm} \mathrm{Hg}$ at $3 \mathrm{mg} / \mathrm{kg} ; 5.3 \pm 0.7 \mathrm{bpm} / \mathrm{mm} \mathrm{Hg}$ at 6 $\mathrm{mg} / \mathrm{kg}$ ). However, $6 \mathrm{mg} / \mathrm{kg}$ rosiglitazone also increased BRG in OR rats without increasing insulin sensitivity, disrupted the correlation between BRG and insulin sensitivity $\left(r^{2}=0.08\right)$, and, in OP and OR rats, elevated BRG relative to insulin sensitivity (analysis of covariance; $P<0.05$ ). Moreover, in OP rats, stimulation of the aortic depressor nerve, to activate central baroreflex pathways, elicited markedly reduced decreases in heart rate and arterial pressure, but these responses were not improved by rosiglitazone. In conclusion, diet-induced obesity impairs BRG via a central mechanism that is related to the concurrent insulin resistance. Rosiglitazone normalizes BRG, but not by improving brain baroreflex processing or insulin sensitivity.

\section{Introduction}

Obesity is associated with multiple serious complications. Within the cardiovascular system, one prominent consequence is dysfunction of the baroreceptor reflex; in particular, baroreflex sensitivity or baroreflex gain (BRG) is decreased (Buñag and Barringer, 1988; Barringer and Buñag, 1989; Grassi et al., 1998; Emdin et al., 2001; Schreihofer et al., 2007). Decreased BRG is a risk factor for the subsequent development of adverse cardiovascular events in patients with type 2 diabetes mellitus (Okada et al., 2010), a common consequence of obesity. Therefore, treatment options are clearly needed.

This work was supported in part by the National Institutes of Health National Heart, Lung, and Blood Institute [Grant HL088552]; and the American Heart Association, Pacific Mountain Affiliate [Grant-in-Aid 09GRNT2060630; Postdoctoral Fellowship 7500041].

Article, publication date, and citation information can be found at http://jpet.aspetjournals.org.

http://dx.doi.org/10.1124/jpet.112.194738
In obese humans, weight loss improves baroreflex function (Grassi et al., 1998; Emdin et al., 2001; Straznicky et al., 2005). However, the success of weight loss as a therapeutic strategy to reverse the pathophysiologic consequences of obesity has been limited (Mark, 2008). As an alternative, pharmaceutical approaches have been investigated. One class of drugs that has been widely prescribed to treat the insulin resistance and hyperglycemia often associated with obesity are thiazolidinediones (TZDs). These drugs activate the nuclear receptor peroxisome proliferator-activated receptor- $\gamma$ $(\mathrm{PPAR}-\gamma)$ to increase insulin sensitivity via multiple mechanisms, including improvement of adipocyte energy storage, production of insulin-sensitizing factors, and actions in adipocytes and macrophages to inhibit production of cytokines, which reduce insulin sensitivity (Berger and Moller, 2002; Duan et al., 2008; Tontonoz and Spiegelman, 2008). Several lines of indirect evidence suggest that TZDs may also enhance baroreflex function in obese individuals by improving insulin sensitivity. First, decreases in BRG and insulin sensitivity are associated in several conditions in addition to

ABBREVIATIONS: BRG, baroreflex gain; TZD, thiazolidinedione; PPAR- $\gamma$, peroxisome proliferator-activated receptor- $\gamma$; DIO, diet-induced obesity; HFD, high-fat diet; OP, obesity prone; OR, obesity resistant; CON, control; MAP, mean arterial pressure; HR, heart rate; ADN, aortic depressor nerve; ROS, reactive oxygen species; ANOVA, analysis of variance; 3-ROSI, $3 \mathrm{mg} / \mathrm{kg}$ rosiglitazone; $6-\mathrm{ROSI}, 6 \mathrm{mg} / \mathrm{kg}$ rosiglitazone. 
obesity, including metabolic syndrome, type II diabetes, hypertension, heart failure, aging, and pregnancy. Second, the improvements in BRG in obese humans after weight loss are related to increases in insulin sensitivity (Grassi et al., 1998; Emdin et al., 2001; Straznicky et al., 2005). Third, pioglitazone was shown to improve baroreflex function and reduce elevated basal sympathetic nerve activity in patients with type 2 diabetes mellitus, soon after myocardial infarction (Yokoe et al., 2012). Finally, our studies of pregnancy (Daubert et al., 2007; Brooks et al., 2010) demonstrated that: 1) the development of insulin resistance and baroreflex impairment are temporally correlated in pregnant rats and rabbits, and 2) treatment of pregnant rabbits with the TZD rosiglitazone normalizes BRG. However, whether TZDs enhance BRG in obese subjects has not been previously investigated.

Therefore, the present experiments tested the hypothesis that rosiglitazone treatment improves obesity-induced baroreflex impairment via increases in insulin sensitivity. We used a rat model of diet-induced obesity (DIO), because it exhibits many of the features of human obesity (Dobrian et al., 2000; Levin and Strack, 2008). We determined whether the magnitudes of insulin resistance and baroreflex dysfunction are correlated in DIO and control $(\mathrm{CON})$ rats and whether treatment of DIO rats with rosiglitazone resolves the baroreflex dysfunction in association with increases in insulin sensitivity. In addition, because previous studies indicate that the baroreflex impairment observed in DIO rats and Zucker obese rats is caused by a depression of the central processing of baroreceptor afferent information (Huber and Schreihofer, 2010; McCully et al., 2012), we tested the hypothesis that rosiglitazone improves baroreflex function by normalizing brain control of the baroreflex. To test this hypothesis, we determined whether the heart rate (HR) and arterial pressure responses to stimulation of the aortic depressor nerve $(\mathrm{ADN})$ are reduced in rats with DIO and whether rosiglitazone enhances these responses.

\section{Materials and Methods}

Male Sprague-Dawley rats (Charles River Laboratories, Inc., Wilmington, MA) were housed individually in cages in a temperaturecontrolled $\left(22 \pm 2^{\circ} \mathrm{C}\right)$ room with a 12-h light/dark cycle. Food and water were provided ad libitum. All procedures were conducted in accordance with the National Institutes of Health's Guide for the Health and Use of Laboratory Animals (Institute of Laboratory Animal Resources, 1996) and approved by the Institutional Animal Care and Use Committee of Oregon Health \& Science University.

\section{DIO Rat Model}

Rats $(\sim 250$ g) were placed on a purified moderately high-fat diet [HFD; 33\% kcal as fat; LabDiet 571R (LabDiet 5001 with $10 \%$ lard; Purina, Richmond, IN)] or a low-fat diet (13.5\% kcal as fat, LabDiet 5001, CON diet). HFD-fed rats diverged into populations with high weight gain [obesity prone (OP)] or weight gain similar to those fed the low-fat control diet [obesity resistant (OR)] (Levin and Strack, 2008). Consistent with significant previous work (Dobrian et al., 2004; Boustany et al., 2005; Levin and Strack, 2008), after 2 weeks on the diet, the top tertile of weight gain was defined as OP, and the bottom tertile was defined as OR. The middle $1 / 3$ rats with intermediate weight gain were not used further.

The OP, OR, and CON rats were then treated with rosiglitazone (GlaxoSmithKline, Uxbridge, Middlesex, UK; 3.0 or $6.0 \mathrm{mg} / \mathrm{kg}$ in vehicle; the amount of rosiglitazone was adjusted every 3 days to account for rat weight gain) or vehicle (1\% carboxymethyl cellulose sodium salt, $0.1 \mathrm{ml} / 100 \mathrm{~g}$; Sigma, St. Louis, MO) daily by gavage until experiments were performed after an additional 2 to 3 weeks. The lower dose of rosiglitazone ( $3 \mathrm{mg} / \mathrm{kg}$ ) was chosen, because it is a frequently used efficacious dose in rats ( Törüner et al., 2004; Khan et al., 2005; Moore et al., 2008; Wang et al., 2010). A higher dose (6 $\mathrm{mg} / \mathrm{kg}$ ) was also tested, because $3 \mathrm{mg} / \mathrm{kg}$ failed to completely normalize insulin sensitivity. It is noteworthy that rosiglitazone exhibits first-order kinetics in rats, such that a doubling of the dose yields a doubling of plasma levels (Wang et al., 2010; Gao and Jusko, 2012).

\section{Experiments in Conscious Rats: Does DIO Impair Baroreflex Function in Association with Insulin Resistance and Are These Changes Reversed by Treatment with Rosiglitazone?}

Surgery. After $\sim 3.5$ weeks on the diets, rats were weighed and anesthetized with $2 \%$ isoflurane in $100 \%$ oxygen. An arterial catheter (PE 50) was aseptically inserted through a small inguinal incision into the femoral artery and advanced into the distal abdominal aorta. In addition, two venous catheters (PE 10) (Becton Dickinson, Sparks, MD) were inserted into the femoral vein and advanced into the distal inferior vena cava. The catheters were tunneled subcutaneously and exteriorized between the scapulae. Catheter patency was maintained by flushing with heparin saline $(100 \mathrm{U} / \mathrm{ml})$ at least three times per week. At least 5 days of recovery were allowed before experimentation.

Experimental Protocols. Insulin sensitivity and BRG were determined in random order with at least 1 day between experiments by using the following protocols.

Baroreflex Curve Generation. Complete baroreflex function curves were produced in conscious rats while they remained in their home cages by using well established, previously published methodology (Xu and Brooks, 1997; Xu et al., 1998). In brief, arterial pressure was increased and decreased by using separate slow intravenous infusions of increasing doses of either phenylephrine or nitroprusside, with each ramp in pressure completed in $\sim 3$ to $5 \mathrm{~min}$. Blood pressure and HR were allowed to return to basal levels before another ramp was initiated. The data were collected by using a Biopac (Goleta, CA) MP100 data acquisition and analysis system sampling at $1000 \mathrm{~Hz}$. The sigmoidal baroreflex relationships between mean arterial pressure (MAP) and HR generated in each experiment were fitted and compared by using the Boltzman equation: $\mathrm{HR}=\left(A_{1}-A_{2}\right) /\left[1+e^{(\mathrm{MAP}-\mathrm{A} 3) / \mathrm{A} 4)}\right]+A_{2}$, where $A_{1}$ equals the maximum $\mathrm{HR}, A_{2}$ equals the minimum $\mathrm{HR}, A_{3}$ equals the MAP at the midpoint between the minimum and maximum HR, and $A_{4}$ is the width or operating range. Maximum baroreflex gain was calculated by dividing the HR range $\left(A_{1}-A_{2}\right)$ by four times the width. Because of technical difficulties (usually catheter failure), it was not possible to assess baroreflex function in all rats.

Insulin Sensitivity Measured using the HyperinsulinemicEuglycemic Clamp Method. Well established procedures were used (DeFronzo et al., 1979; Daubert et al., 2007; Brooks et al., 2010). In brief, the rats were fasted overnight $(\sim 15 \mathrm{~h})$. After a 1 -h equilibration period after connection of the catheters to infusion pumps, arterial blood was collected (five 10- $\mu$ l samples; 5 min apart) for measurement of basal glucose concentration by using a Freestyle Flash blood glucose monitor (Abbott Diabetic Care, Alameda, CA). An additional blood sample $(250 \mu \mathrm{l})$ was collected in vehicle and high-dose rosiglitazone-treated rats for measurement of plasma insulin concentration by radioimmunoassay as described previously (Brooks et al., 2010). A priming dose of human insulin (Novolin R; Novo Nordisk Pharmaceuticals, Inc., Princeton, NJ) was then infused over $10 \mathrm{~min}$, followed by a continuous infusion at $3.0 \mathrm{mU} / \mathrm{kg} /$ $\min (5 \mu \mathrm{l} / \mathrm{min}$ in isotonic saline). Beginning $4 \mathrm{~min}$ later, an intravenous glucose $(50 \%)$ infusion was initiated. Blood samples $(10 \mu \mathrm{l})$ were collected every $10 \mathrm{~min}$ for measurement of glucose concentration, and the glucose infusion rate was adjusted to maintain euglycemia (plasma glucose same as basal). After attaining a constant glucose infusion rate (after $\sim 2 \mathrm{~h}$ ), three final blood glucose levels were measured, $10 \mathrm{~min}$ apart, to document that a steady state had 
been achieved. The steady-state glucose infusion rate was used as an index of insulin sensitivity; higher infusion rates indicated higher insulin sensitivity. To confirm that similar insulin levels were produced by the infusions between groups, a final blood sample $(250 \mu \mathrm{l})$ was collected at the end of the experiment and assayed for human insulin as described previously (Brooks et al., 2010).

Statistical Analysis. One-way ANOVA was used to determine whether conscious OP rats differed from OR and CON rats treated with vehicle (to establish features of the DIO model). Two-way ANOVA [factors were group (OP, OR, and CON) and drug dose (vehicle and 3.0 and $6 \mathrm{mg} / \mathrm{kg}$ rosiglitazone)] evaluated the effects of rosiglitazone treatment. The post hoc Newman Keuls test was used to identify specific within- and between-group differences. Because baroreflex gain increases exponentially, these data were log-transformed before statistical analysis to normalize variability. Data are expressed as mean \pm S.E.M. $P<0.05$ was considered statistically significant.

\section{Experiments in Anesthetized Rats: Does Rosiglitazone Improve Baroreflex Function by Reversing the Effects of Obesity to Impair Central Baroreflex Processing?}

Rats treated with vehicle (2-3 per group) or rosiglitazone (6-9 per group; $6 \mathrm{mg} / \mathrm{kg}$; as described above) were used. In addition, because the responses of vehicle-treated rats were similar to the responses of untreated rats from a previous study (McCully et al., 2012), these data were combined to statistically assess the effects of rosiglitazone.

Surgery. Rats were anesthetized with $\sim 2 \%$ isoflurane in $100 \%$ oxygen, and a femoral arterial catheter and two venous catheters were implanted for the measurement of MAP and HR and intravenous access, respectively. After the completion of the catheter surgery, an intravenous infusion of inactin $(120 \mathrm{mg} / \mathrm{ml}$; Sigma) was administered over $30 \mathrm{~min}$ as the isoflurane was slowly withdrawn. A midline neck incision was made, and the trachea was cannulated (PE 250 trachea cannula; Becton Dickinson) to facilitate spontaneous breathing. Arterial oxygen levels were continuously monitored via a pulse oximeter (Starr Life Sciences, Inc, Oakmont, PA), and, if necessary, adjustments were made in tracheal catheter position to maintain oxygen levels at or above $95 \%$. The left ADN was identified as it joined the superior laryngeal nerve and dissected caudally, and the peripheral end was cut. To remove potential counteracting responses from other arterial baroreceptors inputs, the right $\mathrm{ADN}$ was cut and the carotid sinuses were bilaterally denervated as described previously (McCully et al., 2012). After surgery, a 60-min recovery period was allowed before the experimental protocol was initiated. Rectal body temperature was maintained at $37 \pm 1^{\circ} \mathrm{C}$ throughout by using a heating pad. At the end of the experiment, rats were euthanized with an overdose of pentobarbital sodium (39 mg of Euthasol, intravenously) (Virbac, Fort Worth, TX).

Experimental Protocol: Baroreflex Responses to Electrical Activation of ADN. After dissection, the central end of the ADN was placed on bipolar stainless-steel electrodes and isolated in warm mineral oil. The electrodes were connected to a programmable stimulator (AMPI Master-8; A.M.P.I., Jerusalem, Israel) through a stimulus isolation unit (AMPI ISO-Flex). The frequency and intensity of electrical shocks were varied to activate myelinated A-fibers alone or both A-fibers and nonmyelinated C-fibers. More specifically, lowintensity shocks (1 V) were used to activate only A-fibers, a moderate stimulation ( $3 \mathrm{~V}$ ) was used to maximally activate all A-fibers plus a small contingent of C-fibers, and a high stimulation intensity (20 V) was used to activate both A- and C-fibers (Fan and Andresen, 1998; Fan et al., 1999). At each voltage, 30-s stimulus trains of short duration $(0.1 \mathrm{~ms})$ repeated shocks were administered at 5, 10, 20, 50, and $100 \mathrm{~Hz}$. Reflex responses were measured as peak decreases in MAP and HR (averaged from 5-s stable recordings) relative to $10 \mathrm{~s}$ of baseline data collected just before each stimulation period. Intensity and frequency combinations of the stimuli were applied in random order, with at least 2 min between stimulations.

Statistical Analysis. Three-way ANOVA [factors were group (OP, OR, CON), treatment (rosiglitazone or vehicle/no treatment), and stimulus parameter] was first performed. This analysis revealed highly significant three-way group by treatment by stimulus interactions for both HR $(P<0.001)$ and MAP $(P<0.0001)$, as well as significant $(P<0.05)$ group, stimulus, and group by stimulus and treatment by stimulus interactions for HR and MAP. Specific between- and within-group changes were determined by two-way ANOVA and the post hoc Newman-Keuls test.

\section{Results}

Diet-Induced Obesity Decreases Insulin Sensitivity and Baroreflex Function. After almost 4 weeks on the high-fat diet, OP rats exhibited significantly higher body weight compared with either OR rats or rats ingesting a normal fat diet (Table 1); however, body weight did not differ between $\mathrm{OR}$ and $\mathrm{CON}$ rats. Insulin sensitivity was markedly reduced in OP rats compared with both OR and CON animals (Fig. 1). Human insulin levels were similar (Table 2), confirming that the insulin clamp was equivalent between groups. MAP, HR, and fasting blood glucose and insulin levels did not vary significantly among OP, OR, and CON animals (Tables 1 and 2). BRG (Figs. 2 and 3) was attenuated in OP compared with OR and CON rats. Significant differences in other sigmoidal baroreflex parameters were not observed (Table 3); however, there was a tendency for maximum baroreflex HR to be suppressed in the OP rats $(P=$ 0.08). Among the three groups of vehicle-treated rats, the degree of baroreflex impairment was well correlated to the severity of the insulin resistance (Fig. $4 ; r^{2}=0.49 ; P<$ 0.005).

Rosiglitazone Improves Insulin Sensitivity and Baroreflex Gain in Conscious OP Rats. As expected, rosiglitazone treatment dose-dependently increased insulin sensitivity in OP rats to values exhibited by CON rats (higher rosiglitazone dose; Fig. 1); however, rosiglitazone did not alter insulin sensitivity in OR or CON rats (Fig. 1). Rosiglitazone increased body weight in CON rats, but not in OP or OR rats (Table 1). Nevertheless, body weight remained

TABLE 1

Basal values (conscious rats)

Group numbers are within parentheses.

\begin{tabular}{|c|c|c|c|c|c|c|c|c|c|}
\hline Value & OP & OR & $\mathrm{CON}$ & $\begin{array}{l}\text { OP }+3- \\
\text { ROSI }\end{array}$ & $\begin{array}{l}\text { OR }+3- \\
\text { ROSI }\end{array}$ & $\begin{array}{l}\text { CON + 3- } \\
\text { ROSI }\end{array}$ & $\begin{array}{l}\text { OP }+6- \\
\text { ROSI }\end{array}$ & $\begin{array}{l}\text { OR }+6- \\
\text { ROSI }\end{array}$ & $\begin{array}{l}\text { CON + 6- } \\
\text { ROSI }\end{array}$ \\
\hline Body weight, $\mathrm{g}$ & $484 \pm 15(9)$ & $402 \pm 13 *(8)$ & $380 \pm 9^{*}(8)$ & $491 \pm 8(9)$ & $432 \pm 4^{*}(8)$ & $451 \pm 14^{\dagger}(3)$ & $488 \pm 8(6)$ & $416 \pm 8^{*}(5)$ & $436 \pm 11^{* \dagger}(6)$ \\
\hline $\mathrm{MAP}, \mathrm{mm} \mathrm{Hg}$ & $101 \pm 6(5)$ & $103 \pm 6(5)$ & $99 \pm 3(5)$ & $106 \pm 3(7)$ & $100 \pm 3(5)$ & $97 \pm 2(3)$ & $107 \pm 3(5)$ & $102 \pm 3(5)$ & $110 \pm 5(5)$ \\
\hline $\mathrm{HR}, \mathrm{bpm}$ & $353 \pm 19(5)$ & $364 \pm 4(5)$ & $356 \pm 12(5)$ & $348 \pm 16(7)$ & $334 \pm 7(5)$ & $351 \pm 5(3)$ & $343 \pm 14(5)$ & $344 \pm 7(5)$ & $337 \pm 10(5)$ \\
\hline $\begin{array}{l}\text { Blood glucose, } \\
\text { mg/dl }\end{array}$ & $94 \pm 3(8)$ & $92 \pm 3(8)$ & $89 \pm 2(8)$ & $99 \pm 4(9)$ & $93 \pm 3(8)$ & $89 \pm 6(3)$ & $91 \pm 3(6)$ & $90 \pm 2(5)$ & $93 \pm 2(6)$ \\
\hline
\end{tabular}

*, $P<0.05$ compared with similarly treated OP rats.

${ }^{\dagger}, P<0.05$ significant effect of rosiglitazone within group. 

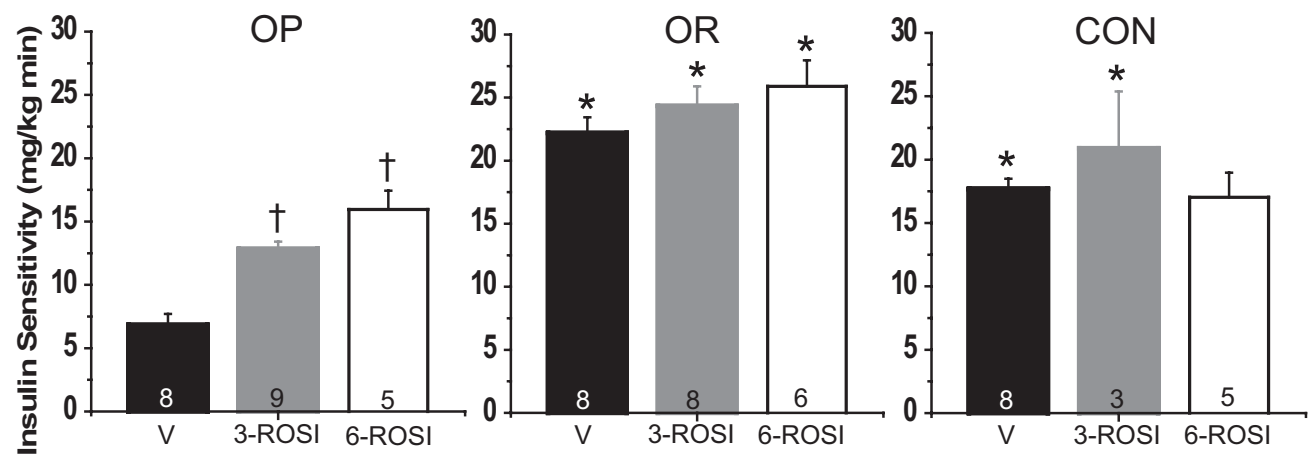

Fig. 1. Insulin sensitivity in OP, OR, and $\mathrm{CON}$ rats treated with vehicle, 3 $\mathrm{mg} / \mathrm{kg}$ rosiglitazone (3-ROSI), and 6 $\mathrm{mg} / \mathrm{kg}$ rosiglitazone (6-ROSI). Twoway ANOVA revealed significant group $(P<0.001)$ and rosiglitazone treatment $(P<0.005)$ effects, as well as a significant group by treatment interaction $(P<0.05)$. *, $P<0.01$ compared with $\mathrm{OP}$, within treatment. $\dagger, P<0.01 \mathrm{com}-$ pared with vehicle, within group. Numbers in bars indicate numbers of rats.

TABLE 2

Plasma insulin levels in fasted rats before (rat insulin) and after (human insulin) the hyperinsulinemic-euglycemic clamp Group numbers are within parentheses.

\begin{tabular}{|c|c|c|c|c|c|c|}
\hline Value & $\mathrm{OP}$ & OR & CON & $\mathrm{OP}+6$-ROSI & $\mathrm{OR}+6$-ROSI & $\mathrm{CON}+6$-ROSI \\
\hline Rat insulin, $\mathrm{ng} / \mathrm{ml}$ & $1.84 \pm 0.48(7)$ & $1.05 \pm 0.37(5)$ & $1.02 \pm 0.31(7)$ & $1.13 \pm 0.15(5)$ & $0.71 \pm 0.09$ & $2.09 \pm 0.60(6)$ \\
\hline Human insulin, $\mu \mathrm{U} / \mathrm{ml}$ & $57.8 \pm 11.6(7)$ & $66.5 \pm 11.0(6)$ & $57.1 \pm 2.9(6)$ & $55.8 \pm 5.5(6)$ & $60.7 \pm 5.6(5)$ & $64.7 \pm 5.0(6)$ \\
\hline
\end{tabular}

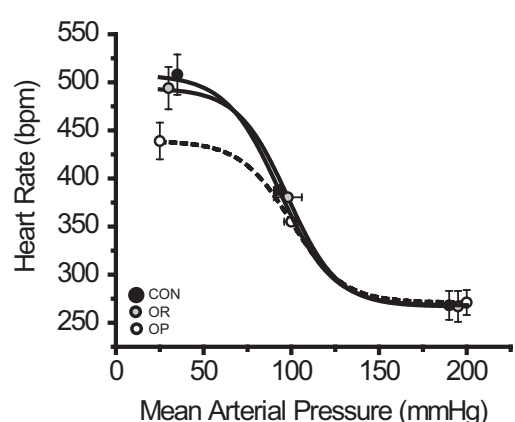

Fig. 2. Sigmoidal baroreflex curves constructed from the means of Boltzman parameters (Table 3) derived from fits of relationships between arterial pressure and heart rate obtained in CON (-), OR (gray-filled circles), and OP rats $(\bigcirc)(n=5$ per group).

elevated in OP rats treated with rosiglitazone compared with similarly treated OR and CON rats (Table 1), and the body weights of OR and CON rats were not different. Rosiglitazone did not significantly influence MAP, HR, or fasting blood glucose and insulin concentrations (Tables 1 and 2).

The low dose of rosiglitazone increased BRG in OP rats but not OR or CON rats (Figs. 3 and 5), such that the differences between OP and OR or CON rats were eliminated. Other baroreflex parameters were not significantly altered (Table 3). The higher dose of rosiglitazone increased BRG further in OP rats; however, the drug also enhanced BRG in OR (but not CON) rats even though insulin sensitivity was not significantly changed (Fig. 3). As a result, the relationship between insulin sensitivity and BRG was shifted upward to a higher gain level in OP, OR, and $\mathrm{CON}$ rats (Fig. 4; analysis of covariance, $P<0.05$ ) such that BRG was significantly elevated for a given level of insulin sensitivity. Moreover, the correlation between these variables was disrupted (Fig. $4 ; r^{2}=0.08$; not significant). These data suggest that the higher dose of rosiglitazone increases BRG by mechanisms unrelated to its effect to improve insulin sensitivity.

HR and MAP Responses to ADN Stimulation Are Impaired in OP Rats, and Rosiglitazone Does Not Reverse This Effect of Obesity. As in the first experimental series, OP rats weighed more than OR or CON rats, and basal HR and MAP were not different between groups
(Table 4). Stimulation of the ADN produced both intensityand frequency-dependent decreases in MAP and HR in all groups (Figs. 6 and $7 ; P<0.05$ ). At stimulus intensities that activate largely A-fibers ( 1 and $3 \mathrm{~V}$ ) the $\mathrm{HR}$ and MAP responses of vehicle-treated OP rats were substantially attenuated compared with similarly treated OR and CON animals (Figs. 6 and 7). At an intensity that activates both A-fibers and C-fibers (20 V) these between-group differences largely disappeared with a significant difference observed only in the $\mathrm{HR}$ responses of OP rats compared with OR rats. In agreement with our previous studies (McCully et al., 2012), these data suggest that obesity impairs the responsiveness of the reflex neuronal pathway mediated by A-fibers at a site beyond the baroreceptors, most likely in the brain.

If rosiglitazone improves baroreflex function by reversing the effects of obesity, then the bradycardic and depressor responses to low/moderate intensity ADN stimulation should improve as well. However, in contrast to this hypothesis, rosiglitazone treatment of OP rats did not enhance responses except at $3 \mathrm{~V}$ (maximal A-fiber intensity) and the highest frequency; the responses of OR rats fed a HFD were also largely unchanged (Figs. 6 and 7). On the other hand, at all intensity levels, the HR responses of rosiglitazone-treated CON rats were significantly reduced; the depressor responses of $\mathrm{CON}$ rats were also attenuated at the low and moderate stimulus intensity (Figs. 6 and 7). These results suggest that in rats fed normal chow rosiglitazone impairs baroreflex responses at a level beyond the baroreceptors (brain or efferent pathway); however, this impairment is neutralized by a HFD.

\section{Discussion}

The purpose of the present study was to develop a model of DIO in rats that causes baroreflex impairment and use this model to test the hypothesis that obesity-induced baroreflex dysfunction can be resolved by rosiglitazone treatment, because of improved insulin sensitivity. The major new findings are that: 1) among rats fed a high-fat diet, those that become obese (OP) exhibit reductions in BRG and insulin sensitivity, and these variables are highly correlated; 2 ) the low dose of rosiglitazone improves insulin sensitivity and BRG in OP rats, but not in $\mathrm{OR}$ or CON rats; 3) the higher dose of 

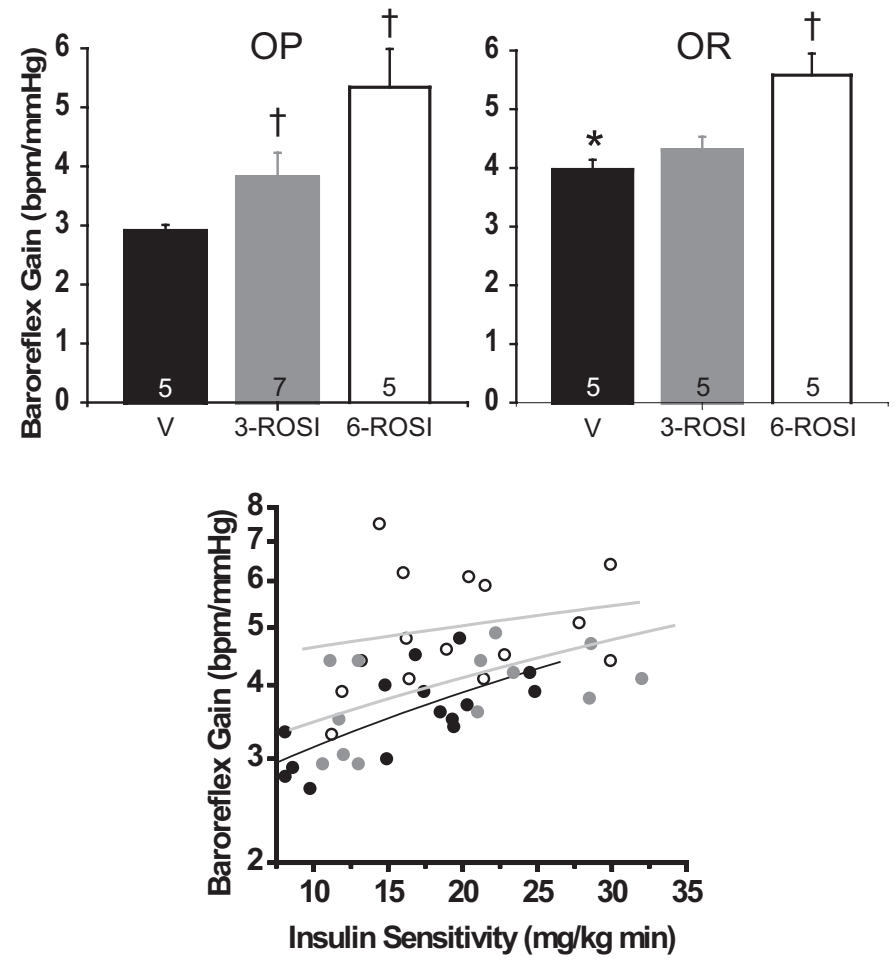

Fig. 4. The degree of baroreflex impairment was well correlated to the severity of the insulin resistance among the vehicle-treated OP, OR, and CON rats $\left(r^{2}=0.49 ; P<0.05\right)$. Rosiglitazone $(3$ or $6 \mathrm{mg} / \mathrm{kg})$ did not alter the slope of this relationship, but the higher rosiglitazone dose increased baroreflex gain relative to the degree of insulin sensitivity (significant increase in intercept; analysis of covariance; $P<0.05)$. $\bullet$, vehicle-treated rats; solid gray circles, rosiglitazone $(3 \mathrm{mg} / \mathrm{kg}) ; \bigcirc$, rosiglitazone $(6 \mathrm{mg} / \mathrm{kg})$.

rosiglitazone produces further increments in BRG in $\mathrm{OP}$ and OR rats relative to insulin sensitivity and disrupts the relationship between BRG and insulin sensitivity; 4) OP rats exhibit impaired HR and MAP responses to ADN stimulation, and rosiglitazone does not restore these responses; and 5) rosiglitazone attenuates depressor and bradycardic responses to ADN stimulation in CON rats fed normal chow. Collectively, these data suggest that although obesity may impair baroreflex function via a mechanism related to the concurrent insulin resistance, rosiglitazone reverses this impairment largely through distinct mechanisms.

Considerable previous research has shown that one of the deleterious cardiovascular consequences of obesity is a decrease in BRG (Buñag and Barringer, 1988; Barringer and Buñag, 1989; Van Vliet et al., 1995; Grassi et al., 1998; Emdin et al., 2001; Schreihofer et al., 2007). Studies in humans suggest that the baroreflex impairment may be related to the accompanying insulin resistance, because body weight reduction improves both BRG gain and insulin sensitivity (Grassi et al., 1998; Emdin et al., 2001). We sought to more directly test this hypothesis by using a rat model of DIO. This obesity model has been used extensively in previous energy balance and cardiovascular investigations and has been shown to exhibit many of the features of human obesity, including a polygenetic basis, activation of the renin-angiotensin and sympathetic nervous systems, and, after a time delay, mild hypertension (Dobrian et al., 2000; Levin and Strack, 2008). A further advantage of the model is that the comparison of OP and OR rats allows identification of mechanisms related to obesity per se, rather than to the high-fat

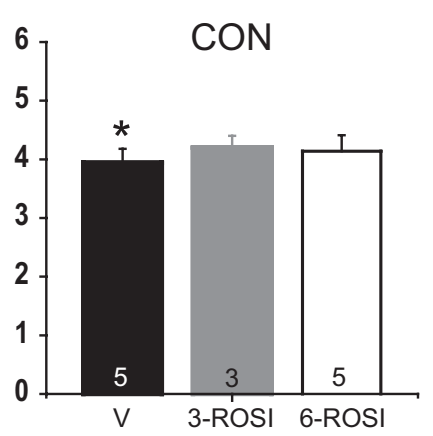

Fig. 3. Baroreflex gain in OP, OR, and CON rats treated with vehicle and 3 and $6 \mathrm{mg} / \mathrm{kg}$ rosiglitazone. Two-way ANOVA revealed significant group $(P<0.05)$ and rosiglitazone treatment $(P<0.0001)$ effects, as well as a group by treatment interaction $(P<$ 0.05). *, $P<0.05$ compared with $\mathrm{OP}$, within treatment. $\dagger, P<0.05$ compared with vehicle, within group. Numbers in bars indicate number of rats.
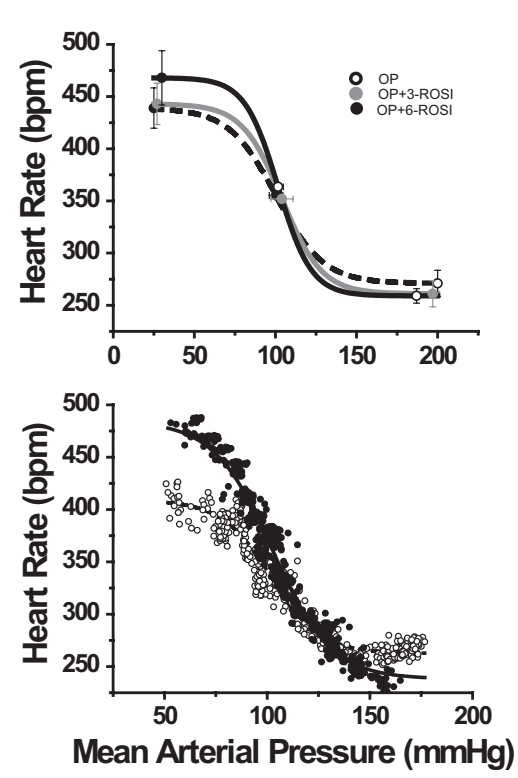

Fig. 5. Top, sigmoidal baroreflex curves constructed from the means of Boltzman parameters (Table 3 ) derived from fits of relationships between arterial pressure and heart rate obtained in OP $(n=5)$, OP + 3-ROSI $(n=7)$, and OP +6 -ROSI $(n=5)$ rats. Bottom, representative experiments showing that treatment of OP rats with $6 \mathrm{mg} / \mathrm{kg}$ rosiglitazone increases baroreflex gain. $\bigcirc$, vehicle-treated OP rats; gray-filled circles, rosiglitazone-treated ( $3 \mathrm{mg} / \mathrm{kg}$ ) OP rats; $\bullet$, rosiglitazone-treated $(6 \mathrm{mg} /$ $\mathrm{kg})$ OP rats.

diet. It is noteworthy that the results of the present study indicate that this DIO model demonstrates another characteristic associated with human obesity, that of impaired HR BRG.

In the present study, the degrees of baroreflex impairment and insulin resistance were well correlated among vehicletreated OP, OR, and CON rats. This result supports the hypothesis that the obesity-induced falls in insulin sensitivity and BRG share common mechanisms. The development of obesity (increased adiposity) seems pivotal, because a highfat diet failed to decrease BRG and insulin sensitivity in OR rats; however, the specific mediator was not identified. Nevertheless, in OP rats, stimulation of the ADN at intensities that activate mostly A-fibers, which largely mediate baroreflex responses near or below resting arterial pressure (Thorén et al., 1977), produced severely attenuated bradycardic and depressor responses. These data point toward a central mechanism. Therefore, rather than an action of insulin resistance per se, an adipocyte-derived factor that contributes to obesity-induced insulin resistance may also impair BRG via a direct or indirect action in the brain. Such factors include cytokines, such as tumor necrosis factor- $\alpha(\mathrm{Yu}$ 
TABLE 3

Baroreflex parameters in OP, OR, and CON rats, either treated or not treated with rosiglitazone Group numbers are within parentheses.

\begin{tabular}{|c|c|c|c|c|c|c|c|c|c|}
\hline Parameter & $\mathrm{OP}(5)$ & OR (5) & CON (5) & $\mathrm{OP}+3$-ROSI (7) & $\underset{3-\text { ROSI (5) }}{\text { OR }}$ & $\underset{3-\mathrm{CON}}{\mathrm{COSI}}+$ & $\underset{\text { 6-ROSI }}{\text { OP }+}$ & $\begin{array}{c}\text { OR }+ \\
\text { 6-ROSI (5) }\end{array}$ & $\underset{\text { 6-ROSI (5) }}{\mathrm{CON}+}$ \\
\hline Maximum, bpm & $439 \pm 19$ & $494 \pm 22$ & $508 \pm 21$ & $443 \pm 20$ & $469 \pm 17$ & $455 \pm 14$ & $468 \pm 26$ & $481 \pm 14$ & $434 \pm 17$ \\
\hline Minimum, bpm & $271 \pm 13$ & $267 \pm 16$ & $268 \pm 15$ & $261 \pm 12$ & $258 \pm 9$ & $255 \pm 16$ & $259 \pm 7$ & $259 \pm 7$ & $240 \pm 18$ \\
\hline Range, bpm & $167 \pm 20$ & $227 \pm 26$ & $240 \pm 19$ & $182 \pm 21$ & $211 \pm 19$ & $200 \pm 20$ & $210 \pm 32$ & $222 \pm 16$ & $194 \pm 22$ \\
\hline $\mathrm{BP}_{50}, \mathrm{~mm} \mathrm{Hg}$ & $100 \pm 4$ & $98 \pm 8$ & $93 \pm 3$ & $104 \pm 3$ & $94 \pm 4$ & $102 \pm 6$ & $102 \pm 3$ & $97 \pm 4$ & $102 \pm 4$ \\
\hline Width, mm Hg & $14.6 \pm 2.3$ & $14.3 \pm 1.4$ & $15.3 \pm 1.2$ & $12.3 \pm 1.5$ & $12.5 \pm 1.4$ & $12.0 \pm 1.1$ & $10.3 \pm 2.0$ & $10.3 \pm 0.8$ & $12.1 \pm 2.0$ \\
\hline
\end{tabular}

TABLE 4

Basal values (anesthetized rats)

Group numbers are within parentheses.

\begin{tabular}{|c|c|c|c|c|c|c|}
\hline Value & $\mathrm{OP}(9)$ & OR (7) & CON (7) & $\mathrm{OP}+6-\mathrm{ROSI}(8)$ & $\mathrm{OR}+6$-ROSI (6) & $\mathrm{CON}+6$-ROSI (6) \\
\hline Body weight, g & $532 \pm 12$ & $440 \pm 10^{*}$ & $437 \pm 11^{*}$ & $536 \pm 11$ & $444 \pm 12^{*}$ & $464 \pm 8^{*}$ \\
\hline $\mathrm{MAP}, \mathrm{mm} \mathrm{Hg}$ & $117 \pm 6$ & $110 \pm 5$ & $117 \pm 5$ & $120 \pm 3$ & $122 \pm 5$ & $128 \pm 4$ \\
\hline $\mathrm{HR}, \mathrm{bpm}$ & $377 \pm 12$ & $387 \pm 17$ & $395 \pm 8$ & $382 \pm 12$ & $376 \pm 7$ & $368 \pm 4$ \\
\hline
\end{tabular}

*, $P<0.05$ compared with similarly treated OP rats.
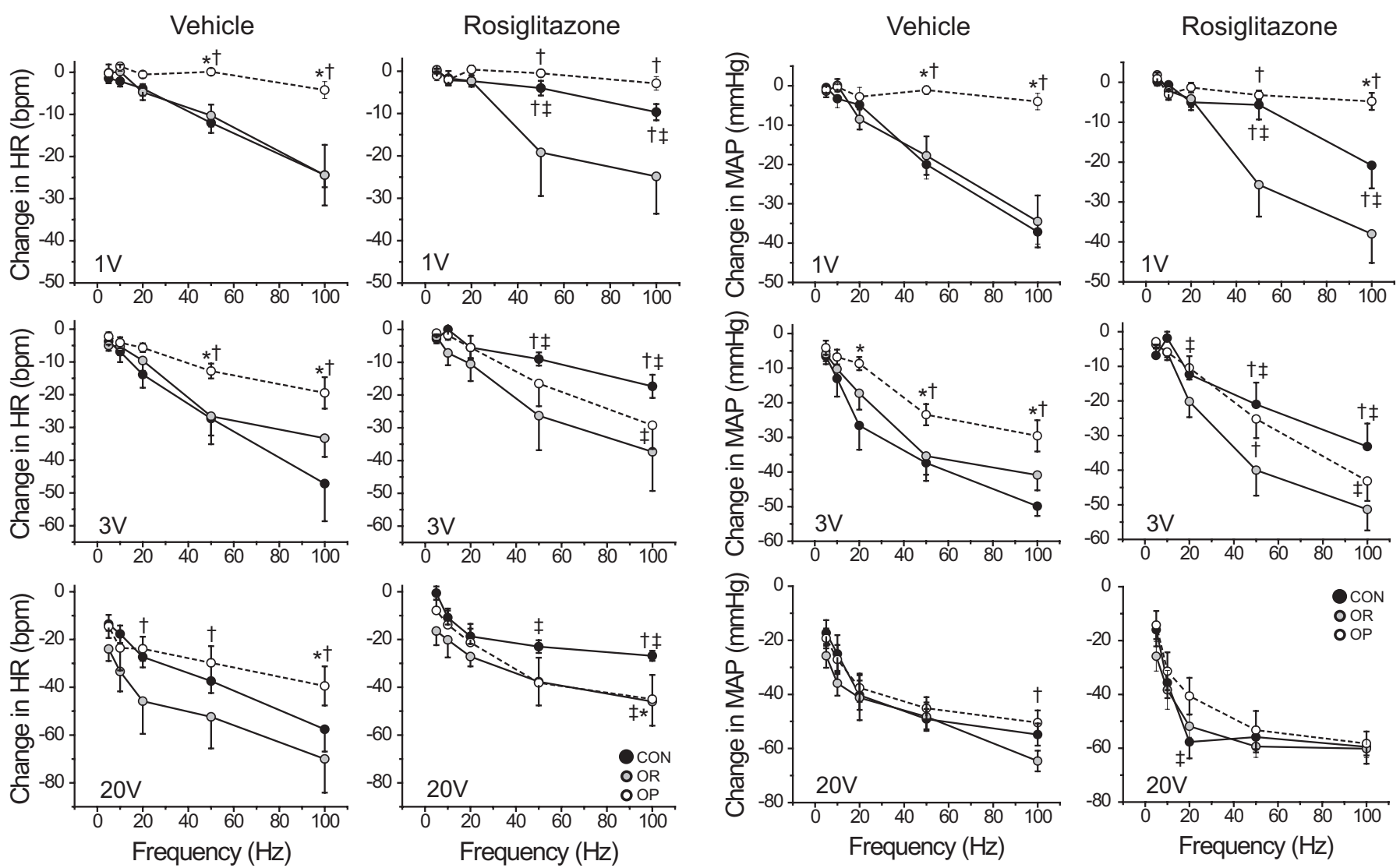

Fig. 6. Bradycardic responses to $\mathrm{ADN}$ stimulation are severely attenuated in OP rats, and rosiglitazone treatment does not restore these responses. *, $P<0.05$ compared with $\mathrm{CON}$. $\dagger, P<0.05$ compared with OR. $\ddagger, P<0.05$, rosiglitazone-treated rats are different from vehicletreated or untreated rats within groups of OP, OR, and CON animals. $\bullet$, CON rats ( $n=6$, rosiglitazone-treated; $n=7$, vehicle-treated or untreated); gray-filled circles, OR rats $(n=6$, rosiglitazone-treated; $n=7$, vehicle-treated or untreated); $\bigcirc$, OP rats $(n=8$, rosiglitazone-treated; $n=9$, vehicle-treated or untreated).

and Ginsberg, 2005; Bastard et al., 2006; Rosen and Spiegelman, 2006; Guggilam et al., 2008) and angiotensin II (Zucker and Liu, 2000; Cassis et al., 2008; Paton et al., 2008).

The finding that rosiglitazone improved BRG in OP rats in association with increases in insulin sensitivity would seem
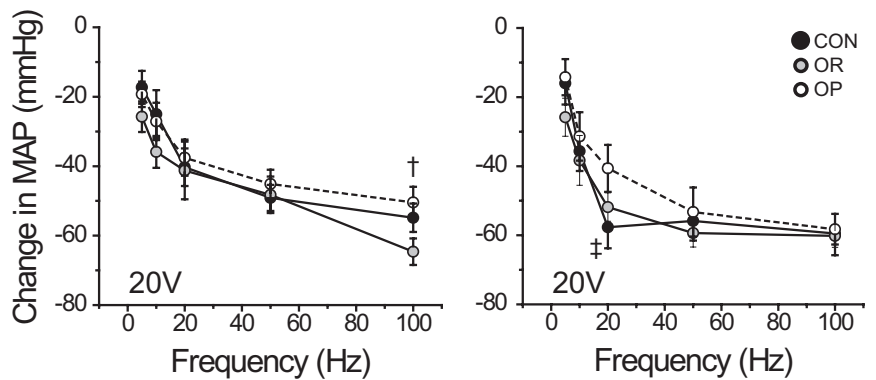

Fig. 7. Depressor responses to ADN stimulation are severely attenuated in OP rats, and rosiglitazone treatment does not restore these responses. $*, P<0.05$ compared with CON. $\dagger, P<0.05$ compared with OR. $\ddagger, P<$ 0.05 rosiglitazone-treated rats are different from vehicle-treated or untreated rats within groups of $\mathrm{OP}, \mathrm{OR}$, and $\mathrm{CON}$ animals. $\bullet$ CON rats ( $n=6$, rosiglitazone-treated; $n=7$, vehicle-treated or untreated), grayfilled circles, OR rats ( $n=6$, rosiglitazone-treated; $n=7$, vehicle-treated or untreated); $\bigcirc$, OP rats $(n=8$, rosiglitazone-treated; $n=9$, vehicletreated or untreated).

to support the hypothesis that this TZD enhances baroreflex function by decreasing the levels or actions of a factor that both contributes to obesity-induced insulin resistance and acts centrally to impair the baroreflex. However, other findings contradict this supposition. First, the higher rosiglita- 
zone dose also increased BRG in OR rats, without altering insulin sensitivity, and elevated BRG relative to insulin sensitivity in OP, OR, and CON rats. Second, rosiglitazone disrupted the correlation between insulin sensitivity and BRG. Third, although the bradycardic responses to ADN stimulation were markedly attenuated in vehicle-treated or untreated OP rats, rosiglitazone increased BRG in conscious OP rats without improving the ADN HR responses; thus, this TZD did not reverse the effect of obesity. These results suggest that rosiglitazone probably increases BRG via a mechanism independent of its effect to increase insulin sensitivity, which parallels the previous observation that mice that express a dominant negative mutation of PPAR- $\gamma$ exhibit hypertension without creating insulin resistance (Tsai et al., 2004).

If not related to improving insulin sensitivity, then how does rosiglitazone enhance baroreflex function? Because PPAR- $\gamma$ is highly expressed in both endothelial and vascular smooth muscle cells (Duan et al., 2008; Marchesi et al., 2008), one possibility is that rosiglitazone improves the baroreflex by enhancing the responsiveness of baroreceptor afferents that are imbedded in the vasculature (Borges et al., 2009). This possibility is consistent with the paired findings that rosiglitazone markedly enhanced BRG in conscious OP and $\mathrm{OR}$ rats, yet did not improve responses to ADN stimulation. Because ADN stimulation bypasses baroreceptor nerve endings, such an effect would be not be evident in these baroreceptor-denervated animals, but would be apparent in intact conscious rats. In support, preliminary studies demonstrate that mice that harbor a dominant negative mutation of PPAR- $\gamma$ in either vascular endothelial cells or vascular smooth muscle exhibit markedly impaired baroreflex sensitivity (Borges et al., 2009; McCully et al., 2011). However, a direct test of this hypothesis requires measurement of the changes in firing of single A-fiber afferents in response to pressure forcings in rosiglitazone-treated rats, because the present results suggest differences in the effect of rosiglitazone on A- versus C-fiber neuronal populations. Alternatively, PPAR- $\gamma$ is expressed in brain (Moreno et al., 2004; Sarruf et al., 2009), and systemic rosiglitazone can enter the brain to activate PPAR- $\gamma$ (Lu et al., 2011; Ryan et al., 2011). Therefore, another possible mechanism is that TZDs, by binding to brain PPAR- $\gamma$, increase HR independently of baroreflex circuitry.

An unexpected finding in the present study was that rosiglitazone treatment of $\mathrm{CON}$ rats significantly attenuated both the depressor and bradycardic responses to ADN stimulation. Whether rosiglitazone acted in the brain or the efferent pathway was not investigated. However, this PPAR- $\gamma$ agonist probably did not impair the response of the heart to efferent autonomic nerves, because 4 to 8 weeks of treatment of rats fed a normal fat diet with a higher dose of rosiglitazone $(8 \mathrm{mg} / \mathrm{kg}$ ) failed to alter the HR responses to propranolol or atropine (Hsieh and Hong, 2008). Likewise, knockout of vascular PPAR- $\gamma$ attenuates vasoconstriction induced by $\alpha$-adrenergic agonists (Halabi et al., 2008; Wang et al., 2009), suggesting that TZDs enhance, rather than inhibit, vascular responses to sympathetic activation. Instead, rosiglitazone may have diminished brainstem processing of arterial baroreceptor afferent inputs. If so, this result may explain why the high dose of rosiglitazone failed to enhance BRG in conscious CON rats; the action of rosiglitazone to improve BRG presumably was counteracted by another central mechanism that impaired this effect. The fact that the diminished ADN responses of $\mathrm{CON}$ rats were not observed in either OP or OR rats suggests that a HFD neutralizes this deleterious action. This possibility is supported by studies demonstrating that the actions of PPAR- $\gamma$ agonists or antagonists (or the loss of PPAR- $\gamma$ in knockout mice) on energy balance are different depending on the presence or absence of a HFD (Diano et al., 2011; Lu et al., 2011; Ryan et al., 2011).

It is well established that TZDs induce weight gain in both humans and experimental animals (Vasudevan and Balasubramanyam, 2004; Lehrke and Lazar, 2005; Diano et al., 2011; Lu et al., 2011; Ryan et al., 2011). As reported previously (Törüner et al., 2004), we found that rosiglitazone increased body weight in CON, but not OR or OP, rats. Brain PPAR- $\gamma$ signaling has been shown to mediate this effect of TZDs in part by stimulating food intake ( $\mathrm{Lu}$ et al., 2011; Ryan et al., 2011). In addition, that work indicates that a HFD, presumably by increasing the levels of fatty acids that act as endogenous ligands for PPAR- $\gamma$, also activates brain PPAR- $\gamma$. Collectively, these findings suggest that rosiglitazone stimulated food intake in CON rats via central activation of hypothalamic PPAR- $\gamma$, but did not increase body weight further in OP rats fed a HFD, because brain PPAR- $\gamma$ was already activated by endogenous ligands. On the other hand, OR rats fail to become obese when exposed to a HFD, because (unlike OP rats) these rats retain sensitivity to the anorexic effects of leptin and insulin (Levin and Strack, 2008). Studies suggest that PPAR- $\gamma$ activation increases food intake by reducing reactive oxygen species (ROS) in proopiomelanocortin neurons, whereas leptin inhibits food intake by increasing ROS (Diano et al., 2011). Therefore, rosiglitazone (or a HFD) may not increase food intake in OR rats, because PPAR- $\gamma$ activation suppresses ROS less, possibly because of a greater sensitivity of pro-opiomelanocortin neurons to the ROS-increasing actions of leptin.

In conclusion, DIO in rats impairs baroreflex control of HR through central mechanisms related to the concurrent insulin resistance. Insulin resistance is a hallmark of many conditions associated with baroreflex dysfunction besides obesity, such as type II diabetes mellitus, hypertension, congestive heart failure, and pregnancy. Therefore, these mechanisms are clearly important to identify, given the propensity of this association and the well established link between decreased BRG and adverse cardiovascular events (Head, 2002; Parati, 2005; Okada et al., 2010). Potential mechanisms include hormones that cause both insulin resistance and act centrally to impair the baroreflex, such as angiotensin II and tumor necrosis factor- $\alpha$. In addition, we show that rosiglitazone treatment improves BRG through mechanisms unrelated to increases in insulin sensitivity. Instead, TZDs may increase BRG by sensitizing baroreceptor afferents and increasing their responsiveness to changes in arterial pressure or by central actions to alter HR independently of brain baroreceptor processing. Future experiments are required to test these hypotheses.

\section{Acknowledgments}

We thank A. Sharen Azar for outstanding technical assistance. 


\section{Authorship Contributions}

Participated in research design: Zhao, McCully, and Brooks.

Conducted experiments: Zhao and McCully.

Performed data analysis: Zhao, McCully, and Brooks.

Wrote or contributed to the writing of the manuscript: Zhao, McCully, and Brooks.

\section{References}

Barringer DL and Buñag RD (1989) Uneven blunting of chronotropic baroreflexes in obese Zucker rats. Am J Physiol Heart Circ Physiol 256:H417-H421.

Bastard JP, Maachi M, Lagathu C, Kim MJ, Caron M, Vidal H, Capeau J, and Feve B (2006) Recent advances in the relationship between obesity, inflammation, and insulin resistance. Eur Cytokine Netw 17:4-12.

Berger J and Moller DE (2002) The mechanisms of action of PPARs. Annu Rev Med 53:409-435.

Borges GR, Morgan DA, Ketsawatsomkron P, Rahmouni K, and Sigmund CD (2009) Tachycardia caused by interference with PPAR- $\gamma$ in vascular smooth muscle in mice is related to autonomic imbalance and baroreflex dysfunction. Hypertension 54:e101.

Boustany CM, Brown DR, Randall DC, and Cassis LA (2005) AT1-receptor antagonism reverses the blood pressure elevation associated with diet-induced obesity. Am J Physiol Regul Integr Comp Physiol 289:R181-R186.

Brooks VL, Mulvaney JM, Azar AS, Zhao D, and Goldman RK (2010) Pregnancy impairs baroreflex control of heart rate in rats: role of insulin sensitivity. Am J Physiol Regul Integr Comp Physiol 298:R419-R426.

Buñag RD and Barringer DL (1988) Obese Zucker rats, though still normotensive, already have impaired chronotropic baroreflexes. Clin Exp Hypertens A 10 (Suppl 1):257-262.

Cassis LA, Police SB, Yiannikouris F, and Thatcher SE (2008) Local adipose tissue renin-angiotensin system. Curr Hypertens Rep 10:93-98.

Daubert DL, Chung MY, and Brooks VL (2007) Insulin resistance and impaired baroreflex gain during pregnancy. Am J Physiol Regul Integr Comp Physiol 292: R2188-R2195.

DeFronzo RA, Tobin JD, and Andres R (1979) Glucose clamp technique: a method for quantifying insulin secretion and resistance. Am J Physiol Endocrinol Metab 237:E214-E223.

Diano S, Liu ZW, Jeong JK, Dietrich MO, Ruan HB, Kim E, Suyama S, Kelly K, Gyengesi E, Arbiser JL, et al. (2011) Peroxisome proliferation-associated control of reactive oxygen species sets melanocortin tone and feeding in diet-induced obesity. Nat Med 17:1121-1127.

Dobrian AD, Davies MJ, Prewitt RL, and Lauterio TJ (2000) Development of hypertension in a rat model of diet-induced obesity. Hypertension 35:1009-1015.

Dobrian AD, Schriver SD, Khraibi AA, and Prewitt RL (2004) Pioglitazone prevents hypertension and reduces oxidative stress in diet-induced obesity. Hypertension 43:48-56.

Duan SZ, Usher MG, and Mortensen RM (2008) Peroxisome proliferator-activated receptor- $\gamma$-mediated effects in the vasculature. Circ Res 102:283-294.

Emdin M, Gastaldelli A, Muscelli E, Macerata A, Natali A, Camastra S, and Ferrannini E (2001) Hyperinsulinemia and autonomic nervous system dysfunction in obesity: effects of weight loss. Circulation 103:513-519.

Fan W and Andresen MC (1998) Differential frequency-dependent reflex integration of myelinated and nonmyelinated rat aortic baroreceptors. Am J Physiol Heart Circ Physiol 275:H632-H640.

Fan W, Schild JH, and Andresen MC (1999) Graded and dynamic reflex summation of myelinated and unmyelinated rat aortic baroreceptors. Am J Physiol Regul Integr Comp Physiol 277:R748-R756.

Gao W and Jusko WJ (2012) Modeling disease progression and rosiglitazone intervention in type 2 diabetic Goto-Kakizaki rats. J Pharmacol Exp Ther 341:617-625.

Grassi G, Seravalle G, Colombo M, Bolla G, Cattaneo BM, Cavagnini F, and Mancia G (1998) Body weight reduction, sympathetic nerve traffic, and arterial baroreflex in obese normotensive humans. Circulation 97:2037-2042.

Guggilam A, Patel KP, Haque M, Ebenezer PJ, Kapusta DR, and Francis J (2008) Cytokine blockade attenuates sympathoexcitation in heart failure: cross-talk between nNOS, AT-1R and cytokines in the hypothalamic paraventricular nucleus. Eur J Heart Fail 10:625-634.

Halabi CM, Beyer AM, de Lange WJ, Keen HL, Baumbach GL, Faraci FM, and Sigmund CD (2008) Interference with PPAR $\gamma$ function in smooth muscle causes vascular dysfunction and hypertension. Cell Metab 7:215-226.

Head GA (2002) Spontaneous baroreflex sensitivity: towards an ideal index of cardiovascular risk. J Hypertens 20:829-831.

Hsieh PS and Hong LZ (2008) Augmentation of nitric oxide is crucial for the time-dependent effects of rosiglitazone on blood pressure and baroreflex function in rats. J Hypertens 26:83-92.

Huber DA and Schreihofer AM (2010) Attenuated baroreflex control of sympathetic nerve activity in obese Zucker rats by central mechanisms. J Physiol 588:15151525

Institute of Laboratory Animal Resources (1996) Guide for the Care and Use of Laboratory Animals 7th ed. Institute of Laboratory Animal Resources, Commission on Life Sciences, National Research Council, Washington, DC

Khan O, Riazi S, Hu X, Song J, Wade JB, and Ecelbarger CA (2005) Regulation of the renal thiazide-sensitive $\mathrm{Na}-\mathrm{Cl}$ cotransporter, blood pressure, and natriuresis in obese Zucker rats treated with rosiglitazone. Am J Physiol Renal Physiol 289: F442-F450.

Lehrke M and Lazar MA (2005) The many faces of PPAR $\gamma$. Cell 123:993-999

Levin BE and Strack AM (2008) Diet-induced obesity in animal models and what they tell us about human obesity, in Neurobiology and Obesity (Harvey $\mathrm{J}$ and Withers DJ eds) pp 164-195, Cambridge University Press, Cambridge, UK.
Lu M, Sarruf DA, Talukdar S, Sharma S, Li P, Bandyopadhyay G, Nalbandian S, Fan W, Gayen JR, Mahata SK, et al. (2011) Brain PPAR- $\gamma$ promotes obesity and is required for the insulin-sensitizing effect of thiazolidinediones. Nat Med 17:618622 .

Marchesi C, Paradis P, and Schiffrin EL (2008) Role of the renin-angiotensin system in vascular inflammation. Trends Pharmacol Sci 29:367-374.

Mark AL (2008) Dietary therapy for obesity: an emperor with no clothes. Hypertension 51:1426-1434.

McCully BH, Brooks VL, and Andresen MC (2012) Diet-induced obesity severely impairs myelinated aortic baroreceptor reflex responses. Am J Physiol Heart Circ Physiol 302:H2083-H2091.

McCully BH, Norton BC, Sigmund CD, and Brooks VL (2011) Dominant-negative mutation of endothelial peroxisome proliferator-activated receptor $\gamma$ (PPAR- $\gamma$ ) impairs spontaneous baroreflex gain in mice. FASEB $J$ 25:841.1.

Moore GB, Pickavance LC, Briscoe CP, Clapham JC, Buckingham RE, and Wilding JP (2008) Energy restriction enhances therapeutic efficacy of the PPARy agonist, rosiglitazone, through regulation of visceral fat gene expression. Diabetes Obes Metab 10:251-263.

Moreno S, Farioli-Vecchioli S, and Cerù MP (2004) Immunolocalization of peroxisome proliferator-activated receptors and retinoid $\mathrm{X}$ receptors in the adult rat CNS. Neuroscience 123:131-145.

Okada N, Takahashi N, Yufu K, Murozono Y, Wakisaka O, Shinohara T, Anan F, Nakagawa M, Hara M, Saikawa T, et al. (2010) Baroreflex sensitivity predicts cardiovascular events in patients with type 2 diabetes mellitus without structural heart disease. Circ J 74:1379-1383.

Parati G (2005) Blood pressure variability: its measurement and significance in hypertension. J Hypertens Suppl 23:S19-S25.

Paton JF, Wang S, Polson JW, and Kasparov S (2008) Signalling across the blood brain barrier by angiotensin II: novel implications for neurogenic hypertension. $J$ Mol Med 86:705-710.

Rosen ED and Spiegelman BM (2006) Adipocytes as regulators of energy balance and glucose homeostasis. Nature 444:847-853.

Ryan KK, Li B, Grayson BE, Matter EK, Woods SC, and Seeley RJ (2011) A role for central nervous system PPAR- $\gamma$ in the regulation of energy balance. Nat Med 17:623-626.

Sarruf DA, Yu F, Nguyen HT, Williams DL, Printz RL, Niswender KD, and Schwartz MW (2009) Expression of peroxisome proliferator-activated receptor- $\gamma$ in key neuronal subsets regulating glucose metabolism and energy homeostasis. Endocrinology 150:707-712.

Schreihofer AM, Mandel DA, Mobley SC, and Stepp DW (2007) Impairment of sympathetic baroreceptor reflexes in obese Zucker rats. Am J Physiol Heart Circ Physiol 293:H2543-H2549.

Straznicky NE, Lambert EA, Lambert GW, Masuo K, Esler MD, and Nestel PJ (2005) Effects of dietary weight loss on sympathetic activity and cardiac risk factors associated with the metabolic syndrome. J Clin Endocrinol Metab 90: 5998-6005.

Thorén P, Saum WR, and Brown AM (1977) Characteristics of rat aortic baroreceptors with nonmedullated afferent nerve fibers. Circ Res 40:231-237.

Tontonoz P and Spiegelman BM (2008) Fat and beyond: the diverse biology of PPARy. Annu Rev Biochem 77:289-312.

Törüner F, Akbay E, Cakir N, Sancak B, Elbeg S, Taneri F, Aktürk M, Karakoc A Ayvaz G, and Arslan M (2004) Effects of PPAR $\gamma$ and PPAR $\alpha$ agonists on serum leptin levels in diet-induced obese rats. Horm Metab Res 36:226-230.

Tsai YS, Kim HJ, Takahashi N, Kim HS, Hagaman JR, Kim JK, and Maeda N (2004) Hypertension and abnormal fat distribution but not insulin resistance in mice with P465L PPAR $\gamma$. J Clin Invest 114:240-249.

Van Vliet BN, Hall JE, Mizelle HL, Montani JP, and Smith MJ Jr (1995) Reduced parasympathetic control of heart rate in obese dogs. Am J Physiol Heart Circ Physiol 269:H629-H637.

Vasudevan AR and Balasubramanyam A (2004) Thiazolidinediones: a review of their mechanisms of insulin sensitization, therapeutic potential, clinical efficacy, and tolerability. Diabetes Technol Ther 6:850-863.

Wang N, Symons JD, Zhang H, Jia Z, Gonzalez FJ, and Yang T (2009) Distinct functions of vascular endothelial and smooth muscle PPAR $\gamma$ in regulation of blood pressure and vascular tone. Toxicol Pathol 37:21-27.

Wang X, Liu X, Zhan Y, Lavallie ER, Diblasio-Smith L, Collins-Racie L, Mounts WM, Rutkowski JL, Xu X, Goltsman I, et al. (2010) Pharmacogenomic, physiological, and biochemical investigations on safety and efficacy biomarkers associated with the peroxisome proliferator-activated receptor- $\gamma$ activator rosiglitazone in rodents: a translational medicine investigation. J Pharmacol Exp Ther 334:820-829.

Xu L and Brooks VL (1997) Sodium intake, angiotensin II receptor blockade, and baroreflex function in conscious rats. Hypertension 29:450-457.

Xu L, Collister JP, Osborn JW, and Brooks VL (1998) Endogenous ANG II supports lumbar sympathetic activity in conscious, sodium deprived rats: role of area postrema. Am J Physiol Regul Integr Comp Physiol 275:R46-R55.

Yokoe H, Yuasa F, Yuyama R, Murakawa K, Miyasaka Y, Yoshida S, Tsujimoto S, Sugiura T, and Iwasaka T (2012) Effect of pioglitazone on arterial baroreflex sensitivity and sympathetic nerve activity in patients with acute myocardial infarction and type 2 diabetes mellitus. J Cardiovasc Pharmacol 59:563-569.

Yu YH and Ginsberg HN (2005) Adipocyte signaling and lipid homeostasis: sequelae of insulin-resistant adipose tissue. Circ Res 96:1042-1052.

Zucker IH and Liu JL (2000) Angiotensin II-nitric oxide interactions in the control of sympathetic outflow in heart failure. Heart Fail Rev 5:27-43.

Address correspondence to: Dr. Virginia L. Brooks, Department of Physiology and Pharmacology, L-334, Oregon Health \& Science University, 3181 SW Sam Jackson Park Road, Portland, OR 97239. E-mail: brooksv@ohsu.edu 